\title{
Zirconium Carbide Oxidation: Maltese Cross Formation and Interface Characterization
}

\author{
Claudia Gasparrini ${ }^{1}$ (D) Renaud Podor $^{2}$. \\ Denis Horlait $^{1,3} \cdot$ Richard Chater $^{4}$ - William Edward Lee ${ }^{1}$
}

Received: 11 November 2016/Published online: 2 December 2016

(C) The Author(s) 2016. This article is published with open access at Springerlink.com

\begin{abstract}
Oxidation of dense hot-pressed $\mathrm{ZrC}$ specimens from 1073 to $1473 \mathrm{~K}$ was investigated using an in situ technique: HT-ESEM. Cuboid specimens were monitored on the surface and on edges and corners during oxidation in order to understand the influence of crack formation and propagation on the Maltese cross shape development of the oxide. The oxidation mechanism comprised three steps: (1) delamination of sample edges, (2) crack formation at corners and (3) crack propagation towards the inner core and formation of microcracks parallel to the interface that increase the accessible surface area followed by a drastic volume expansion. The microcrack pattern is found to be repetitive as if a cyclic debonding of the interface occurred. Characterization of the interface by TEM and HRTEM revealed
\end{abstract}

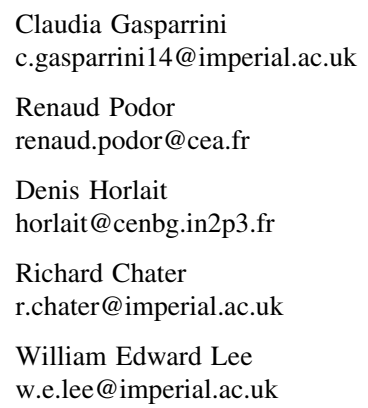

1 Centre for Nuclear Engineering (CNE) \& Department of Materials, Imperial College London, South Kensington Campus, London SW7 2AZ, UK

2 Institut de Chimie Séparative de Marcoule, UMR 5257 CEA/CNRS/UM/ENSCM, Site de Marcoule, BP17171, 30207 Bagnols-Sur-Cèze, France

3 CNRS, Centre D’Etudes Nucléaires de Bordeaux-Gradignan, UMR 5797, Chemin Du Solarium, 33175 Gradignan, France

4 Department of Materials, Imperial College London, South Kensington Campus, London SW7 2AZ, UK 
the interface between $\mathrm{ZrC}$ and $\mathrm{ZrO}_{2}$ to comprise a $2 \mu \mathrm{m}$ thick amorphous carbon matrix with $\mathrm{ZrO}_{2}$ nanocrystals embedded in it.

Keywords Zirconium carbide $\cdot$ Oxidation $\cdot$ Maltese cross $\cdot$ HT-ESEM

\section{Introduction}

Zirconium carbide $(\mathrm{ZrC})$ is a candidate ceramic for use in ultra-high temperature applications in hypersonic vehicles [1,2] and in the nuclear industry as an inert matrix fuel or as a structural component in tristructural-isotropic (TRISO) [3, 4] fuel particles for Generation IV reactors. Its advantageous properties include high temperature stability in non-oxidizing atmospheres, high melting point (around $3700 \mathrm{~K})$ [5], and high thermal conductivity $\left(20 \mathrm{Wm}^{-1} \mathrm{~K}^{-1}\right.$ at $\left.300 \mathrm{~K}\right)$ [6]. However, zirconium carbide readily oxidizes from relatively low temperatures (as low as $653 \mathrm{~K}$ according to Shimada and Ishii) [7]. A comprehensive understanding of the oxidation mechanism and kinetics of $\mathrm{ZrC}$ is thus important for predicting its behaviour under accident scenarios in which $\mathrm{ZrC}$ would come in contact with air at high temperature. Data reported in previous studies consider $\mathrm{ZrC}$ oxidation in the form of powder [7-9], sintered pellet [10-13] and single crystal [14, 15] under many different conditions. What is understood and well reported regarding $\mathrm{ZrC}$ oxidation is that $\mathrm{ZrC}$ is susceptible to rapid oxidation at high temperatures ( $\mathrm{T} \gtrsim 870 \mathrm{~K}$ ) [16] and that the mechanism of formation of the oxide occurs with the typical "Maltese cross" shape [17], previously reported for other carbides and borides of group IV, $\mathrm{V}$, VI of the transitions metals [18-21]. However, an overall model describing the controlling mechanism is difficult to achieve as $\mathrm{ZrC}$ oxidation is strongly affected by a range of parameters and previous studies show limited experimental data in overlapping parameter ranges [16]. The parameters that mainly influence the oxidation behaviour of $\mathrm{ZrC}$ are temperature, oxygen partial pressure, stoichiometry $\left(\mathrm{ZrC}_{1-x}\right)$ and samples intrinsic properties derived from their preparation method (i.e. porosity, impurity nature and level $[16,22]$ ). An overall understanding of the $\mathrm{ZrC}$ oxidation mechanism is also difficult to achieve as discrepancies in activation energy values in experiments performed over the same range of temperature show that a competition between diffusion-controlled and phase boundary controlled reaction occurs [23, 24]. Kuriakose and Margrave studied the oxidation of electron beam melted $\mathrm{ZrC}$ at $100 \mathrm{kPa}$ of $\mathrm{O}_{2}$. For $827-925 \mathrm{~K}$ their measured kinetics were found to follow linear rates throughout the temperature range studied while at higher temperatures (973-1123 K) a destructive oxidation was reported. Shimada et al. [25]. investigated the microstructure of the oxide after oxidation experiments from 773 to $873 \mathrm{~K}$ on $\mathrm{ZrC}$ single crystals. Observations of cross sections revealed two oxide regions. Zone 1 was described as a pore free and compact intermediate region enriched in carbon with crystallites of cubic zirconium oxide $\left(\mathrm{c}-\mathrm{ZrO}_{2}\right)$ that grew parabolically to $2-3 \mu \mathrm{m}$ thick. This acted as a diffusion barrier against oxidation. Zone 2 was instead described as a carbon-poor layer of cracked zirconium oxide which grew linearly above Zone 1 . A mechanistic model of the oxidation of $\mathrm{ZrC}$ was first proposed by Rama Rao and Venugopal [23] and then by Katoh et al. [16]. 
The proposed models suggest as initial stage the formation of an oxycarbide $\left.\mathrm{ZrO}_{x} \mathrm{C}_{(1-x)}\right)$ layer at the interface [7, 16, 23]. This intermediate layer however has never been experimentally observed. The present work thus aims to provide information and understanding on the oxidation mechanism of hot pressed specimens of $\mathrm{ZrC}$ from 1073 to $1473 \mathrm{~K}$ with a particular attention to the nature of the intermediate layer and to the mechanism of formation of the oxide. The peculiarity of this work is the use of state-of-the-art characterisation techniques: in situ high-temperature environmental scanning electron microscopy (HT-ESEM) for investigations on the Maltese cross formation and TEM and HRTEM for the characterization of the $\mathrm{ZrC} / \mathrm{ZrO}_{2}$ interface.

\section{Experimental Procedures}

High density ( $>96 \%$ of the theoretical density, TD) disc-shaped $\mathrm{ZrC}$ pellets were produced by hot pressing $\mathrm{ZrC}$ commercial powder $(3-5 \mu \mathrm{m}, 90 \%<8 \mu \mathrm{m}$ with $0.2 \%<\mathrm{Hf}<2 \%$, Grade B, H.C. Starck, Karlsruhe, Germany) in a vacuum hot press furnace (FCT Systeme, GmbH, Germany). The $\mathrm{ZrC}$ powder was inserted in a $40 \mathrm{~mm}$ graphite die and hot pressed at 2123 or $2273 \mathrm{~K}$ in argon atmosphere for $1 \mathrm{~h}$ under $50 \mathrm{MPa}$ of uniaxial pressure. These disc-shaped specimens were firstly ground and polished with 120, 500 and 1200 MD-Piano Struers cloths and then machined into specimens of known dimension: $15 \mathrm{~mm} \times 8 \mathrm{~mm} \times 5 \mathrm{~mm}$ cuboids (Set A), $10 \mathrm{~mm}$ side cubes (Set B) and $4 \mathrm{~mm} \times 4 \mathrm{~mm} \times 0.5 \mathrm{~mm}$ cuboids (Set C) via electrical discharge machining method (EDM). These samples were used for oxidation tests and characterisation of the intermediate layer between the oxide and the carbide (Set A), quenching studies (Set B) and in situ HT-ESEM oxidation studies for characterisation of the Maltese cross formation mechanism (Set C). Specimens had a density within the range of $6.48-6.58 \mathrm{~g} / \mathrm{cm}^{3}$ evaluated with the Archimedes method from the average of three measurements so that the theoretical density is about $96.2 \% \mathrm{TD}$ for samples hot-pressed at $2123 \mathrm{~K}$ (Set A) and 97.1-99.2\% TD for those hot-pressed at $2273 \mathrm{~K}$ (Sets B and C). Before oxidation experiments, coupons were washed in an ultrasonic bath of acetone and dried at $110{ }^{\circ} \mathrm{C}$ overnight. Oxidation experiments on Set A were performed in a furnace at $1073 \mathrm{~K}$ in air atmosphere, Set B was used for oxidation in air in a chamber lift furnace from 1073 to $1373 \mathrm{~K}$ with fixed quenching times at 15, 30, 60, 120, 240, 360 and 480 min so that different stages of oxidation could be followed while Set C was used for oxidation at 1073 and $1473 \mathrm{~K}$ in a HT-ESEM (Quanta 200 FEG ESEM) equipped with a hot stage in a 2 mbar oxygen atmosphere. This analysis was performed at the Marcoule Institute for Separative Chemistry (ICSM), Marcoule, France. Samples were first heated in the HT-ESEM chamber to the desired temperature under vacuum atmosphere, then when the required temperature was reached, a 2 mbar oxygen flux was introduced into the chamber. Sample corners were constantly monitored by taking pictures every second during the first $5 \mathrm{~min}$ of the experiments, every $5 \mathrm{~s}$ after $5 \mathrm{~min}$ up to $2 \mathrm{~h}$ and every $30 \mathrm{~s}$ after $2 \mathrm{~h}$ up to $16 \mathrm{~h}$. All images were recorded with the same field of view corresponding to $1050 \mu \mathrm{m}$ 
squared. General procedure and details about the HT-ESEM platform can be found in refs [26-28].

Microstructural characterisation of the carbide-oxide interface on partly-oxidised cross sections was performed at Imperial College London. First observations were made with a SEM (LEO Gemini 1525 FEG-SEM, Zeiss, Jena, Germany) using secondary electron imaging (SEI) equipped with an energy dispersive $\mathrm{x}$-ray spectrometer (EDX) for chemical analysis (INCA, Oxford Instruments, Oxford, UK). Nanoscale analysis of the intermediate layer between the carbide and the oxide was performed with a transmission electron microscope (TEM) (2000FX, JEOL, Tokyo, Japan) and a High Resolution-TEM (JEOL JEM 2100F). Samples for TEM analysis were prepared with the FIB (focussed ion-beam) technique (Helios Nanolab 600, FEI Company). Selected area diffraction (SAD) patterns were indexed by matching the $d_{h k 1}$ values with reference patterns. A SingleCrystal software (version 2.3 CrystalMaker Software Limited, Oxfordshire, UK) simulation was performed to recheck the assigned indexes. These characterisation techniques were performed on samples cooled to room temperature. The results therefore show the structure of the oxide after it had undergone several modifications due to volume changes. Indeed $\mathrm{ZrO}_{2}$ undergoes transitions between monoclinic, tetragonal and cubic polymorphs within the range of temperatures used in this study [29, 30]. In particular the volume change occurring in the transition from the tetragonal to the monoclinic structure during cooling is estimated to be about $+3 \mathrm{vol} . \%[31,32]$. To investigate the role of crack formation in the oxide development as a Maltese cross excluding any effect of cracking due to volume change when cooling, the in situ experiment on a central area of the sample surface and on sample corner's in the HT-ESEM is required.

\section{Results and Discussion}

\section{Maltese Cross Formation}

ESEM analysis (Fig. 1) of sample surfaces shows the transition from a compact oxide to a cracked oxide layer. Cracks are mostly generated along the grain boundaries and these initiate and propagate up to approximately $50 \mathrm{~min}$ after the sample starts being exposed to oxygen atmosphere.

Once cracks propagate at the surface along the grain boundaries, the oxide growth rate accelerates. This was evident during the HT-ESEM experiment as the corrections required to maintain the surface of interest at the set working distance and therefore with the right focus were more important. This is due to a considerable volume expansion. The oxidation at this stage becomes severe and the typical Maltese cross of the oxide develops (see Figs. 2, 3).

When the oxide growth is severe, it is accompanied by shrinkage of the unoxidised carbide core as revealed in Fig. 2 (bottom). This picture shows specimens (Set B) oxidized at $1273 \mathrm{~K}$ in air in a chamber lift furnace with quenching at a certain time: specimens in the top row show their oxide layer still intact while specimens at the bottom row have had their oxide layer removed so that the evolution of the unoxidised carbide core can be followed. For oxidation times 

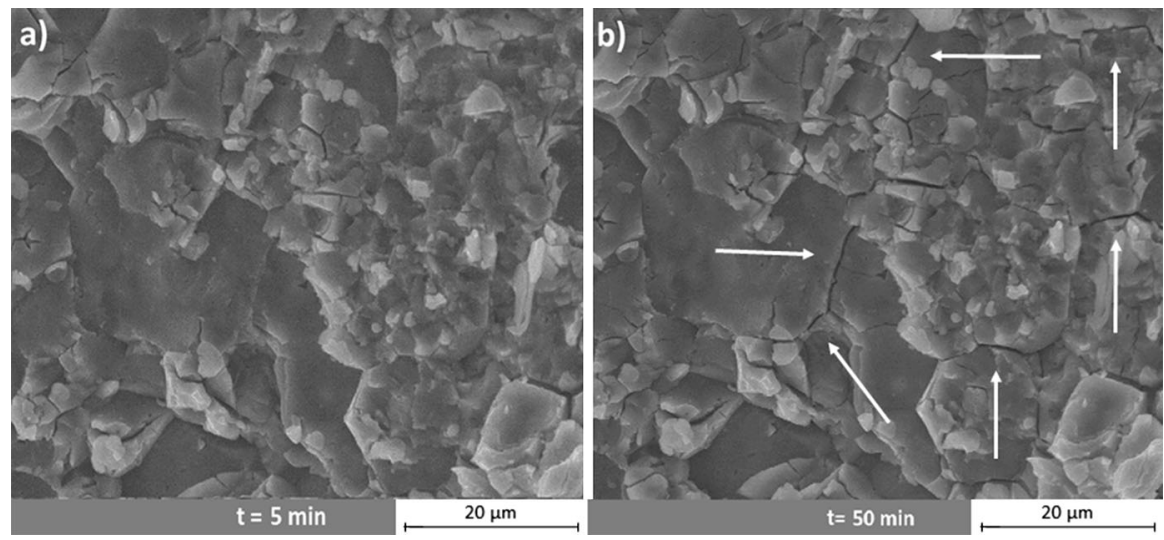

Fig. 1 a SEI of $\mathrm{ZrC}$ sample (Set C) exposed to 2 mbar of oxygen at $1073 \mathrm{~K}$ after 5 min of oxygen flow. b SEI of $\mathrm{ZrC}$ exposed to 2 mbar of oxygen at $1073 \mathrm{~K}$ after $50 \mathrm{~min}$ of oxygen flow: crack formation along the boundaries

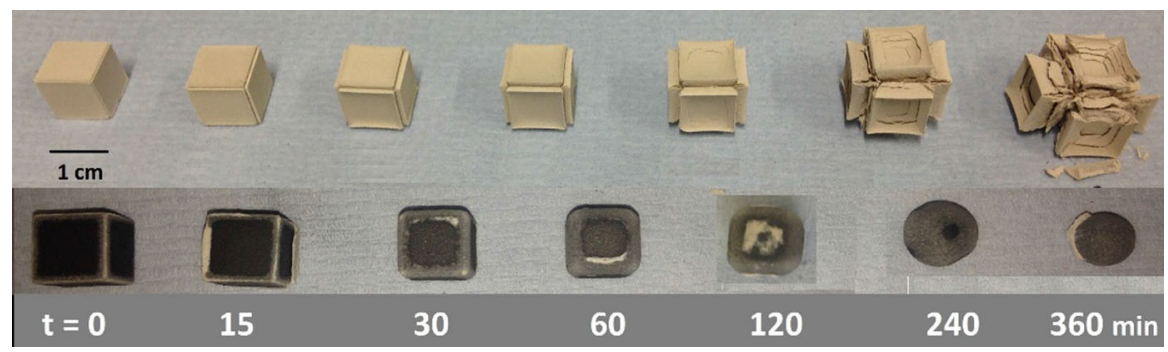

Fig. 2 Image of $\mathrm{ZrC}$ specimens (Set B) oxidised at $1273 \mathrm{~K}$ in a chamber lift furnace: specimens at the top show the evolution of the oxide layer with time, specimens at the bottom had their oxide layer removed so that the evolution of the unoxidised carbide core can be seen
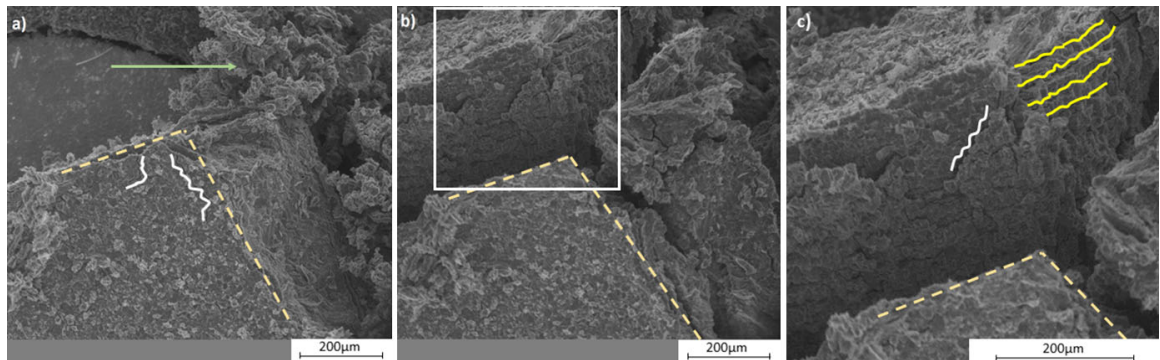

Fig. 3 SEI of a $\mathrm{ZrC}$ sample (Set C) oxidised at $1073 \mathrm{~K}$ for a $40 \mathrm{~min}$ and b $480 \mathrm{~min}$ and high magnification SE image of the area highlighted with a white box in Fig. $3 \mathrm{~b}$ c details on the monitored corner of the sample (dotted segments), the initial delamination followed by spallation at the corner (arrow), the appearance of large cracks at the corner (a) (white lines) and from the corner towards the inner core (c) (white line) and microcracks that run parallel to the carbide-oxide interface (c) (yellow lines) (Color figure online) 
over $2 \mathrm{~h}$ the initial cubic inner carbide core assumes a rounded then spherical shape whilst the oxide develops with the characteristic Maltese cross shape (Fig. 2). The change in shape of the unoxidised carbide core from cubic to spherical suggests that oxidation proceeds quicker at sample corners, therefore the monitoring of a sample corner in the HT-ESEM is performed (see Figs. 3, 4).

In Figs. 3 and 4, we report the monitoring during oxidation at 2 mbar $\mathrm{O}_{2}$ and $1073 \mathrm{~K}$ at the corners of two Set $\mathrm{C}$ samples. These two experiments allowed the identification of three different steps/phenomena occurring during the oxidation of $\mathrm{ZrC}$ and which contribute to the formation of the Maltese cross shape for the oxide.

The first step/phenomenon is the delamination and spallation of the edges and corners of the oxidized sample seen in Fig. 3a) (indicated with an arrow) but much better evidenced in Fig. 4b). This occurs before the centres of the faces, monitored in Fig. 1, gets heavily cracked and is thus still compact (Fig. 1a). This indicates that the corner is the first area to fail already suggesting the oxidation process is faster at
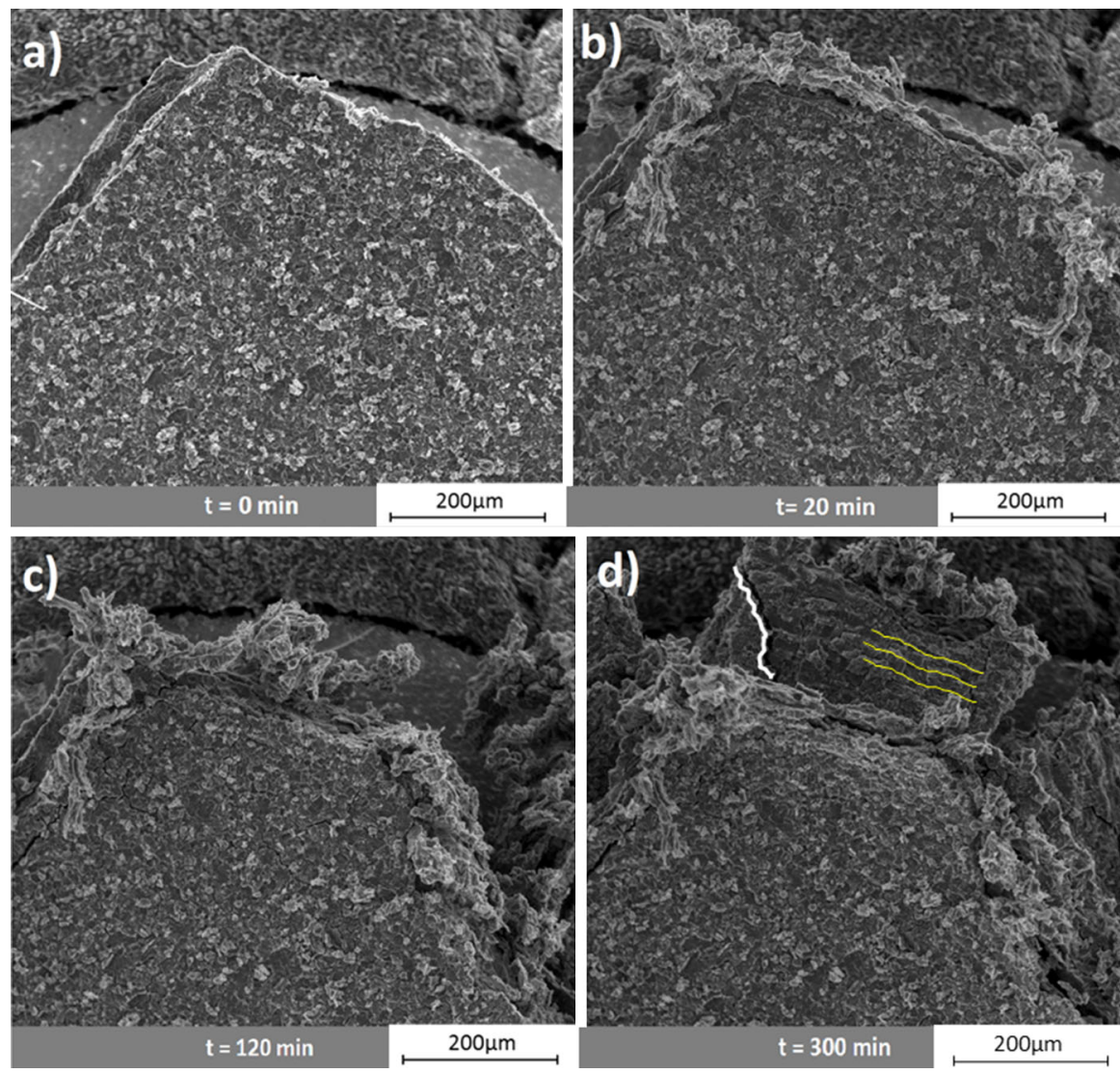

Fig. 4 Sequence of SEI on Set C sample oxidized at $1073 \mathrm{~K}$ in a 2 mbar oxygen atmosphere, a sample at the beginning of the oxidation: time 0 ; $\mathbf{b}$ delamination of the oxide layer at edges and spalling off; $\mathbf{c}$ crack formation and opening up of corners; $\mathbf{d}$ cracks development towards the inner core (highlighted with a white line) and microcrack propagation 
corners. Spallation of the first layer of the oxide leaves the surfaces at the corner exposed to faster oxide penetration. The ensuing rapid stress build-up at the corners is presumably responsible for the following steps.

The second phenomenon involves crack formation at the corner (white lines in Fig. 3a) and from the corner towards the inner core (crack pointed by the white lines in Figs. 3c, 4d) thus the opening of the corners and edges due to the build-up of stresses. The cracking of the corners offers a route for the oxygen to reach the inner unoxidised $\mathrm{ZrC}$ core promoting the characteristic Maltese cross shape.

The development of microcracks at the corners parallel to the oxide/carbide interface, as evidenced in Fig. 3c (yellow lines), constitute the third and last observed phenomenon. For experiments performed in air atmosphere they eventually become visible by the naked eye as can be observed in Fig. 2 (samples on the top oxidised at 120, 240 and $360 \mathrm{~min}$ ). The formation of these microcracks obviously further increases the accessible reactive area in the initial corner regions, therefore accelerating drastically the oxidation reaction and the associated volume increase at the corners/edges compared to the face centres. Proof of the volume expansion occurring because of the two cracking phenomenon is indirectly given in Fig. 3c: when the choice is made to keep the upper part in focus, the bottom part of the surface (bottom of Fig. 3c) gets blurred thus out of focus and this is obviously due to a drastic change in height between the two separated parts of the original corner.

Another interesting observation was made about the cracks parallel to the oxide/carbide interface: these microcracks occur in a repetitive pattern as the distance between each layer (yellow lines in Fig. 3c) is approximately $20 \mu \mathrm{m}$ thick. This repetitive pattern suggests that these cracks are generated cyclically and they are due to debonding of the interface between the carbide and the oxide. The carbide/oxide interface characterization is also part of the present report and is discussed in the following section. Thanks to the use of the HT-ESEM it was possible to show that cracking is not due to stress build-up related to phase transformation during cooling but instead is due to the volume expansion associated with the oxidation of the carbide.

\section{Oxide Layer and Interface Characterisation}

Characterisation of the oxide layer and the carbide/oxide interface was performed on a polished sample's cross section annealed at $1073 \mathrm{~K}$ in air atmosphere for $1 \mathrm{~h}$ (Set A). Figure 5 shows an SEI of the interface between the carbide and the porous oxide layer. The intermediate region appears to be a dense pore-free layer of about $20 \mu \mathrm{m}$ thickness. EDX analysis on this intermediate region shows the presence of carbon, oxygen and zirconium agreeing with the suggestion by Shimada et al. of an oxycarbide [33] compound. Nanoscale analysis on the interface was done on a FIB milled TEM sample cut out from this region (see Fig. 5b). Bright-field TEM imaging (Fig. 6a) reveals that the compact interface region seen by SEM (Fig. 5a, b) is made of three zones, numbered 1,2 and 3 in Fig. 6a. Zone 1 is indexed as the [ $\left.\begin{array}{lll}1 & 1 & 0\end{array}\right]$ zone axis of $\mathrm{ZrC}$ and the $\mathrm{SAD}$ pattern from the circled area of Zone 1 is shown in Fig. 6b. Zone $3 \mathrm{SAD}$ pattern reveals that this region is polycrystalline with 

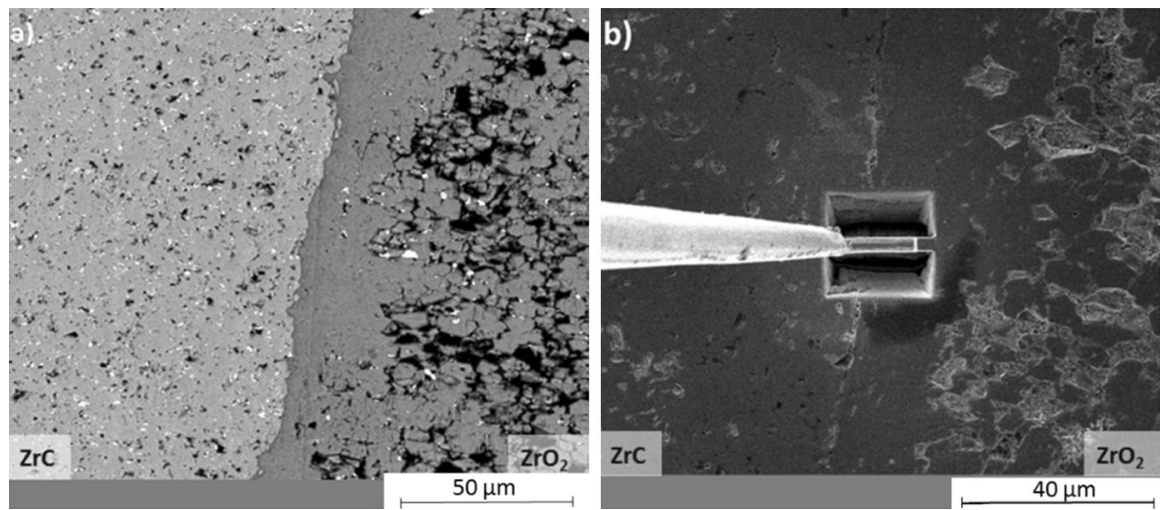

Fig. 5 a SEI of $\mathrm{ZrC}$ polished cross section (sample annealed at $1073 \mathrm{~K}$ for $60 \mathrm{~min}$ ); b FIB milling on the dense intermediate layer between the carbide and the oxide for TEM sample preparation
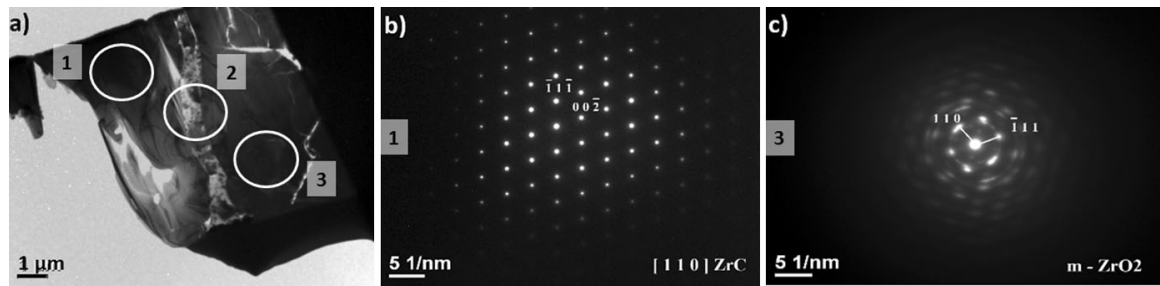

Fig. 6 a BF TEM image of the intermediate region sample; $\mathbf{b}$ and $\mathbf{c}$ SAD patterns of Zone 1 and 3 of TEM specimen in Fig. 6a
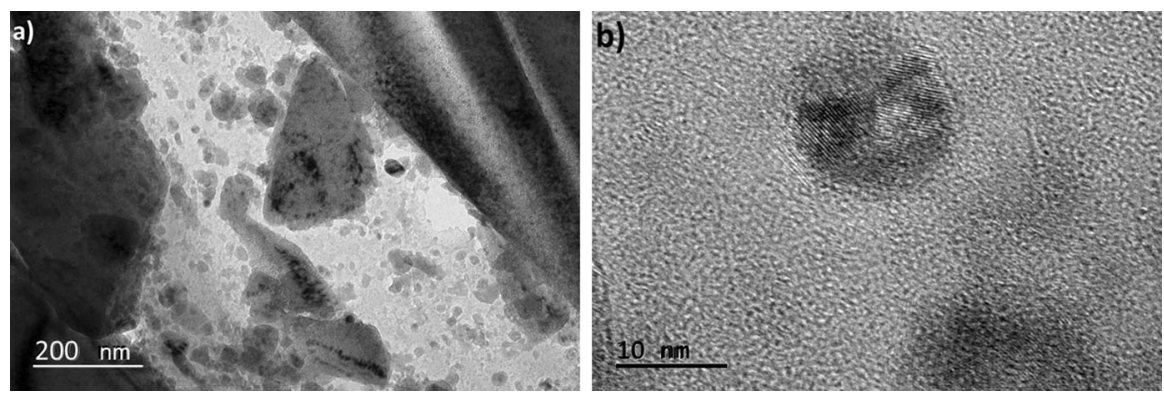

Fig. 7 a High magnification image of the intermediate layer numbered 2 in Fig. 6a; b high resolution details of the intermediate region between $\mathrm{ZrC}$ and $\mathrm{m}-\mathrm{ZrO}_{2}$ showing an amorphous carbon matrix containing nanocrystals of $\mathrm{ZrO}_{2}(<5 \mathrm{~nm})$

SAD rings of Fig. 6c matching monoclinic $\mathrm{ZrO}_{2}$. Zone 2 was not characterised via this TEM as the smallest selected area aperture available was bigger than the area of interest. HRTEM characterisation of the Zone 2 region seen in Fig. 6a is shown in Fig. 7a. It revealed a previously undetected thin region $(<2 \mu \mathrm{m})$ present at the interface between the $\mathrm{ZrC}$ and the dense polycrystalline $\mathrm{m}-\mathrm{ZrO}_{2}$ layer. This layer surprisingly consists of an amorphous carbon matrix containing $\mathrm{ZrO}_{2}$ nanocrystals 
$(<5 \mathrm{~nm})$. The nanoscale analysis performed with TEM and HRTEM thus proved that the approximately $20 \mu \mathrm{m}$ wide dense and pore free intermediate layer present at the carbide/porous oxide interface and observed by SEM (Figs. 5a, b) is actually made of two regions: on the carbide surface a thin intermediate layer $(<2 \mu \mathrm{m})$ predominantly comprised of amorphous carbon and $\mathrm{ZrO}_{2}$ nanocrystals along with a larger layer $(\sim 20 \mu \mathrm{m})$ of polycrystalline $\mathrm{m}-\mathrm{ZrO}_{2}$. Investigations performed on the sample intermediate dense region seen in Fig. 5a with a focused ion beam instrument coupled with a secondary ions mass spectrometer (FIB-SIMS) confirmed this area to be made of a thin layer $(<2 \mu \mathrm{m})$ attached to the carbide region and $\mathrm{ZrO}_{2}$. Chemical analysis performed by SIMS and EDX on a $100 \mu \mathrm{m}$ squared sputtered region across the interface showed the carbon content dropping to zero $5 \mu \mathrm{m}$ away from $\mathrm{ZrC}$ and oxygen rising from zero to $66 \%$ atomic value proving that stoichiometric $\mathrm{ZrO}_{2}$ is formed.

\section{Concluding Remarks}

Formation of the Maltese cross shape of the oxide during oxidation of dense hot pressed $\mathrm{ZrC}$ specimens has been investigated with an in situ technique: HT-ESEM. The mechanism of formation of the oxide during air oxidation comprises three steps: delamination of the first layer of the oxide at the corners and edges of the sample-crack formation at the corners due to build-up of stresses-crack propagation towards the inner unoxidised carbide core followed by propagation of microcracks parallel to the carbide-oxide interface. The propagation of these microcracks increases the accessible reactive area for the oxygen to access. This is followed by a drastic development of the oxide which assumes the typical Maltese cross shape. The microcracks parallel to the interface follow a cyclical pattern as their separation is approximately $20 \mu \mathrm{m}$ in thickness. This infers a cyclic oxidation prior to debonding of the interface while at temperature, cracking is therefore not due to phase transformation stresses during cooling. To further understand the crack formation at corners and debonding of the interface a finite element modelling code will be used to simulate the volume expansion of the oxide during heating revealed by the HT-ESEM experiments. Investigation using TEM and HRTEM advanced techniques revealed that the dense $20 \mu \mathrm{m}$ thick $\mathrm{ZrO}_{2}$ layer found at the surface of $\mathrm{ZrC}$ during its oxidation also comprises a previously undetected and unreported $2 \mu \mathrm{m}$ layer made of an amorphous carbon matrix with nanocrystals of $\mathrm{ZrO}_{2}$ embedded in it.

The present study is ongoing and will be extended by performing TOF/SIMS analysis through the oxide layer with $\mathrm{O}^{18}$ as a marker in oxidation experiments. These additional actions are undertaken to provide solid arguments on $\mathrm{ZrC}$ oxidation behaviour and the results will be given in a future publication.

Acknowledgements The authors are grateful to the EPSRC DISTINCTIVE (Decommissioning, Immobilisation and Storage soluTIons for NuClear wasTe InVEntories) Consortium for their financial support of this project (EPSRC Industrial Case Award EP/M507428/1 Grant and the DISTINCTIVE EP/ L014041/1 Grant). We also thank Dr Robert Harrison for discussion, Dr Mahmoud Ardakani for help with HRTEM analysis and Dr Ecaterina Ware and Garry Stakalls for help with sample preparation. 
Open Access This article is distributed under the terms of the Creative Commons Attribution 4.0 International License (http://creativecommons.org/licenses/by/4.0/), which permits unrestricted use, distribution, and reproduction in any medium, provided you give appropriate credit to the original author(s) and the source, provide a link to the Creative Commons license, and indicate if changes were made.

\section{References}

1. A. Paul, D. D. Jayaseelan, S. Venugopal, E. Zapata-Solvas, J. Binner, B. Vaidhyanathan, A. Heaton, P. Brown and W. E. Lee, Bulletin American Ceramic Society 91, 22 (2012).

2. E. Wuchina, E. Opila, M. Opeka, W. Fahrenholtz and I. Talmy, Electrochemical Society Interface 16, 30 (2007).

3. W. E. Lee, M. Gilbert, S. T. Murphy and R. W. Grimes, Journal of the American Ceramic Society 96,2005 (2013).

4. K. Minato, T. Ogawa, K. Fukuda, H. Nabielek, H. Sekino, Y. Nozawa and I. Takahashi, Journal of Nuclear Materials 224, 85 (1995).

5. H. F. Jackson, D. D. Jayaseelan, D. Manara, C. P. Casoni and W. E. Lee, Journal of the American Ceramic Society 94, 3561 (2011).

6. H. F. Jackson, and W. E. Lee, in Comprehensive Nuclear Materials, ed. R. J. M. Konings, Vol. 2 (Elsevier Ltd., Amsterdam, 2012), pp 339-72.

7. S. Shimada and T. Ishii, Journal of the American Ceramic Society 73, 2804 (1990).

8. A. S. Shevchenko, R. A. Lyutikov, R. A. Andrievskii and V. A. Terekhova, Soviet Powder Metallurgy and Metal Ceramics 19, 48 (1980).

9. R. W. Bartlett, M. E. Wadsworth and I. B. Cutler, Transactions of the Metallurgical Society of AIME 227, 467 (1963).

10. K. R. Janowski, R. D. Carnahan and R. C. Rossi, Static and Dynamic Oxidation of ZrC. Report no TDR-669(6250-10)-3, (Aerospace Corp., El Segundo, 1966).

11. J. B. Berkowitz-Mattuck, Journal of the Electrochemical Society 114, 1030 (1030).

12. R. F. Voitovich and E. A. Pugach, Soviet Powder Metallurgy and Metal Ceramics 12, 916 (1973).

13. D. Gozzi, M. Montozzi and P. L. Cignini, Solid State Ionics 123, 11 (1999).

14. A. Bellucci, D. Gozzi, T. Kimura, T. Noda and S. Otani, Surface and Coatings Technology 197, 294 (2005).

15. A. K. Kuriakose and J. L. Margrave, Journal of the Electrochemical Society 111, 827 (1964).

16. Y. Katoh, G. Vasudevamurthy, T. Nozawa and L. L. Snead, Journal of Nuclear Materials 441, 718 (2013).

17. E. J. Opila, and N. S. Jacobson, in Ceramics Science and Technology, eds. R. Riedel and I-W. Chen, Vol. 4 (Wiley-VCH Verlag GmbH \& Co. KGaA, Weinheim, 2013), pp 1-93.

18. D. Sciti, L. Silvestroni, S. Guicciardi, D. D. Fabbriche and A. Bellosi, Journal of Material Research 24,2056 (2009).

19. L. Silvestroni and D. Sciti, Advances in Materials Science and Engineering 2010,1 (2010).

20. M. Desmaison-Brut, N. Alexandre and J. Desmaison, Journal of the European Ceramic Society 17, 1325 (1997).

21. D. Mattia, M. Desmaison-Brut, S. Dimovski, Y. Gogotsi and J. Desmaison, Journal of the European Ceramic Society 25, 1789 (2005).

22. X.-M. Hou and K.-C. Chou, Journal of Alloys and Compounds 509, 2395 (2011).

23. G. A. Rama Rao and V. Venugopal, Journal of Alloys and Compounds 206, 237 (1994).

24. L. C. Dufour, J. Simon and P. Barret, Comptes Rendus de l'Académie des Sciences-Series C 265, 171 (1967).

25. S. Shimada, M. Nishisako, M. Inagaki and K. Yamamoto, Journal of the American Ceramic Society 78, 41 (1995).

26. N. Clavier, R. Podor, L. Deliere, J. Ravaux and N. Dacheux, Materials Chemistry and Physics 137, 742 (2013).

27. D. Horlait, L. Claparede, F. Tocino, N. Clavier, J. Ravaux, S. Szenknect, R. Podor and N. Dacheux, Journal of Materials Chemistry A 2, 5193 (2014). 
28. R. Podor, D. Pailhon, J. Ravaux and H.-P. Brau, Microscopy and Microanalysis 21, 307 (2015).

29. R. W. Harrison and W. E. Lee, Journal of the American Ceramic Society 9,1 (2015 ).

30. R. N. Patil and E. C. Subbarao, Acta Crystallographica Section A 26, 535 (1970).

31. Y. Moriya and A. Navrotsky, The Journal of Chemical Thermodynamics 38, 211 (2006).

32. R. N. Patil and E. C. Subbarao, Journal of Applied Crystallography 2, 281 (1969).

33. S. Shimada, M. Inagaki and M. Suzuki, Journal of Material Research 11, 2594 (1996). 